\title{
Original
}

\section{Diagnostic significance of PAX8 in thyroid squamous cell carcinoma}

\author{
Ayana Suzuki ${ }^{1)}$, Mitsuyoshi Hirokawa ${ }^{2)}$, Nami Takada ${ }^{1)}$, Miyoko Higuchi ${ }^{1)}$, Naoki Yamao ${ }^{1)}$, Seiji Kuma ${ }^{2)}$, \\ Tsutomu $\mathrm{Daa}^{3)}$ and Akira Miyauchi ${ }^{4)}$ \\ 1) Department of Laboratory, Kuma Hospital, Kobe 650-0011, Japan \\ 2) Department of Diagnostic Pathology, Kuma Hospital, Kobe 650-0011, Japan \\ 3) Department of Diagnostic Pathology, Faculty of Medicine, Oita University, Yufu 879-5593, Japan \\ 4) Department of Surgery, Kuma Hospital, Kobe 650-0011, Japan
}

\begin{abstract}
Most types of thyroid carcinomas express PAX8 transcription factor; however, whether thyroid squamous cell carcinoma (SCC) also expresses PAX8, currently remains unknown. We herein examined the immunoreactivity of PAX8 in SCC of thyroidal and extrathyroidal origin, and discussed the diagnostic significance of PAX8. We immunohistochemically examined specimens from 11 SCC, 22 papillary thyroid carcinoma (PTC), 8 anaplastic thyroid carcinoma (ATC), and 2 mucoepidermoid carcinoma (MEC) cases as well as 5 uterine cervical SCC, 5 esophageal SCC, and 5 pulmonary SCC cases. The rates of PAX8-positive SCC, PTC, ATC, and MEC were 90.9\%, 90.9\%, 75.0\%, and 100\%, respectively. Two PAX8-negative PTC cases were cribriform variants. No uterine cervical, esophageal, or pulmonary SCC specimen reacted with PAX8 antibody. Thyroid transcription factor-1 (TTF-1) was positive in $9.1 \%$ and $95.5 \%$ of SCC and PTC cases, respectively, but negative in all ATC and MEC cases. These results demonstrate that PAX8 staining is useful for distinguishing between primary thyroid SCC and invasion or metastasis from extrathyroidal SCC. We recommend using an immunohistochemical panel of antibodies to PAX8 and TTF-1 to confirm a diagnosis of primary thyroid carcinoma.
\end{abstract}

Key words: Thyroid, Immunohistochemistry, PAX8, Squamous cell carcinoma, Esophagus

PRIMARY SQUAMOUS CELL CARCINOMA (SCC) of the thyroid is microscopically composed entirely of carcinoma cells with squamous differentiation, and its incidence is very low [1]. SCC was previously shown to be a component of papillary thyroid carcinoma (PTC) or anaplastic thyroid carcinoma (ATC) [1]. These carcinomas are aggressive and frequently invade the surrounding organs, including the larynx, esophagus, and trachea. It is important to distinguish primary thyroid SCC from an invasion of SCC arising in the adjacent organs, because the prognosis of the former is worse than that of the latter [2]. However, difficulties are associated with preoperatively confirming a diagnosis of primary thyroid SCC in fine needle aspiration cytology or core needle biopsy samples. The presence of PTC or ATC may indicate primary thyroid SCC. Immunohistochemically, SCC

Submitted Apr. 17, 2015; Accepted Aug. 19, 2015 as EJ15-0226 Released online in J-STAGE as advance publication Sep. 9, 2015 Correspondence to: Ayana Suzuki, Department of Laboratory, Kuma Hospital, 8-2-35, Shimoyamate-dori, Chuo-ku, Kobe, Hyogo 6500011, Japan. E-mail: suzuki01@kuma-h.or.jp cells generally express high-molecular-weight cytokeratin (HMW-CK), p63, and p53 [3]; however, an organspecific marker has not yet been identified.

Paired box gene 8 (PAX8) is a transcription factor that is essential for embryonic development of the kidney, Müllerian organs, and thyroid [4]. The expression of PAX8 was recently detected immunohistochemically in carcinomas arising in these organs [4]. In the thyroid, PAX8 was shown to be immunopositive for PTC, follicular thyroid carcinoma (FTC), poorly differentiated thyroid carcinoma (PDTC), medullary thyroid carcinoma (MTC), and even ATC $[5,6]$. To the best of our knowledge, it has yet to be determined whether thyroid SCC expresses PAX8. Therefore, we herein examined the immunoreactivity of PAX8 in thyroidal and extrathyroidal SCC, and discussed the diagnostic significance of PAX8.

\section{Materials and Methods}

We searched the pathology report database of 8,365 cases of thyroid carcinoma that were operated on in 
Table 1 Primary antibodies used in immunostaining and antigen retrieval methods

\begin{tabular}{lllllc}
\hline Antibody & Clone & \multicolumn{1}{c}{ Vendor } & \multicolumn{1}{c}{ Location } & Antigen retrieval & Dilution \\
\hline PAX8 & polyclonal & Protein Tech & Chicago, IL, USA & Heat (pH6) & $1: 200$ \\
TTF-1 & 8G7G3/1 & Dako & Carpinteria, CA, USA & Heat (pH6) & $1: 100$ \\
p63 & 4 A4 & Nichirei Biosciences & Tokyo, Japan & Heat (pH9) & $(-)$ \\
HMW-CK & $34 \beta E 12$ & Dako & Carpinteria, CA, USA & Protein K & $1: 3$ \\
\hline
\end{tabular}

TTF-1, thyroid transcription factor-1; HMW-CK, high-molecular-weight cytokeratin.

Table 2 Immunohistochemical results of thyroid carcinomas

\begin{tabular}{lrccc}
\hline & PAX8 & TTF-1 & p63 & HMW-CK \\
\hline SCC $(11)$ & $10(90.9 \%)$ & $1(9.1 \%)$ & $11(100 \%)$ & $10(90.9 \%)$ \\
PTC (22) & $20(90.9 \%)$ & $21(95.5 \%)$ & $1(4.5 \%)$ & $0(0 \%)$ \\
SM $(10)$ & $8(80.0 \%)$ & $5(50.0 \%)$ & $8(80.0 \%)$ & $9(90.0 \%)$ \\
Morules $(7)$ & $1(14.3 \%)$ & $0(0 \%)$ & $0(0 \%)$ & $0(0 \%)$ \\
ATC $(8)$ & $6(75.0 \%)$ & $2(25.0 \%)$ & $5(62.5 \%)$ & $5(62.5 \%)$ \\
MEC $(2)$ & $2(100 \%)$ & $0(0 \%)$ & $2(100 \%)$ & $2(100 \%)$ \\
\hline
\end{tabular}

SCC, squamous cell carcinoma; PTC, papillary thyroid carcinoma; SM, squamous metaplasia; ATC, anaplastic thyroid carcinoma; MEC, mucoepidermoid carcinoma; TTF-1, thyroid transcription factor-1; HMW-CK, highmolecular-weight cytokeratin.

Kuma Hospital between 2008 and 2014, and 11 cases that had the SCC component in at least a part of the tumor were extracted. The diagnosis of SCC required apparent carcinoma cells with squamous differentiation, which was identified by the presence of keratinization, an intercellular bridge, or stratification. We reviewed these cases, and confirmed that 3 out of 11 of them were composed of only the SCC component. The remaining eight cases were associated with PTC or ATC. We also extracted 22 PTC, 8 ATC, and 2 mucoepidermoid carcinoma (MEC) cases in the same manner. Ten and seven out of the 22 PTC cases were PTC with squamous metaplasia and the cribriform variant with morula, respectively. The remaining cases were conventional PTC. We also examined 5 uterine cervical SCC, 5 esophageal SCC, and 5 pulmonary SCC cases that were collected from the pathology files of Oita University Hospital.

We performed immunostaining using 3- $\mu$ m-thick, formalin-fixed, paraffin-embedded tissue. The primary antibodies used in the immunostaining and antigen retrieval methods were shown in Table 1. Staining was carried out using the Leica Bondmax system (Leica Microsystems IL) and Bond refine kit (Leica Microsystems, IL) according to the manufacturer's recommendation. The positive decision criteria of immunostaining were defined as cases with more than $10 \%$ carcinoma cells expressing moderate or strong positive staining [7].

\section{Results}

\section{Primary thyroid carcinomas}

38 (88.4\%) out of 43 thyroid carcinoma cases were positive for PAX8, and in $35(81.4 \%)$ of them more than $50 \%$ of carcinoma cells showed the immunopositivity for the antibody. Ten $(90.9 \%)$ out of 11 SCC cases showed nuclear positivity for PAX8 (Fig. 1A) (Table 2). In some cases, the foci, in which the nuclei and cytoplasm were both equally stained, were observed. We regarded this phenomenon as false positive. Twenty (90.9\%) out of 22 PTC cases, six (75.0\%) out of eight ATC cases, and all two MEC cases were immunopositive for PAX8. In SCC and ATC cases that were judged as PAX8-negative, there were no carcinoma cells reacting with the antibody. Papillary carcinomas invariably showed diffuse and strong positivity for PAX8, except for cribriform variant PTC, which showed an inhomogeneous staining pattern. Two PAX8-negative PTC cases were the cribriform variant. Squamous metaplasia was positive for the antibody (Fig. 2A). The PAX8-positive rate of morules in cribriform variant PTC was $14.3 \%$.

One $(9.1 \%)$ out of 11 SCC cases and 21 (95.5\%) out of 22 PTC cases were positive for thyroid transcription factor-1 (TTF-1) (Fig. 1B). The SCC case and 2 ATC cases without PAX8 expression were both included among TTF-1-negative cases. The TTF-1-positive rate in the foci of squamous metaplasia was 50.0\% (Fig. 2B). 


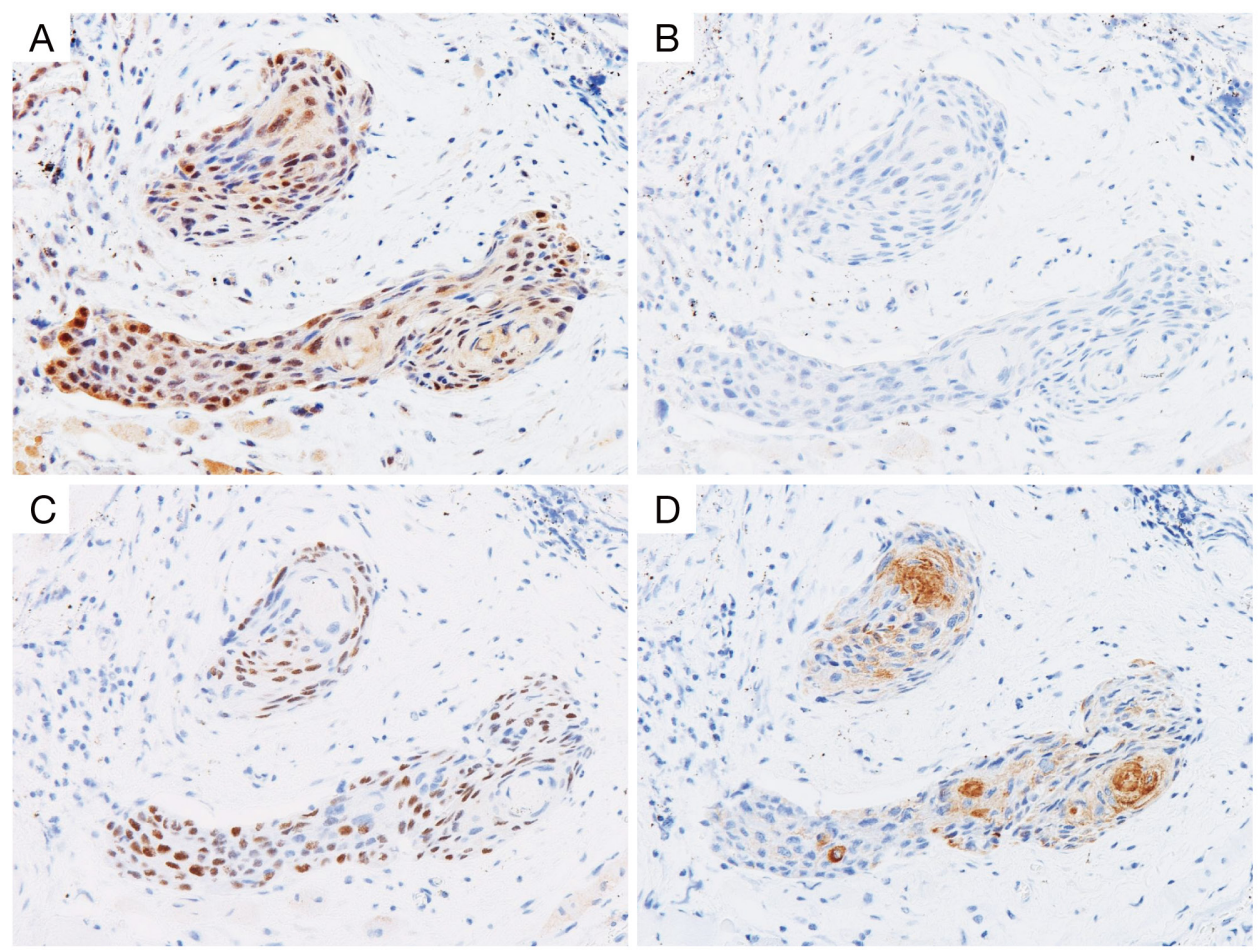

Fig. 1 Squamous cell carcinoma. Most carcinoma cells were moderately positive for PAX8 (A). TTF-1 was negative (B). Carcinoma cells located at the periphery of the nests were positive for p63 (C). High-molecular-weight cytokeratin reacted with keratinized carcinoma cells (D).
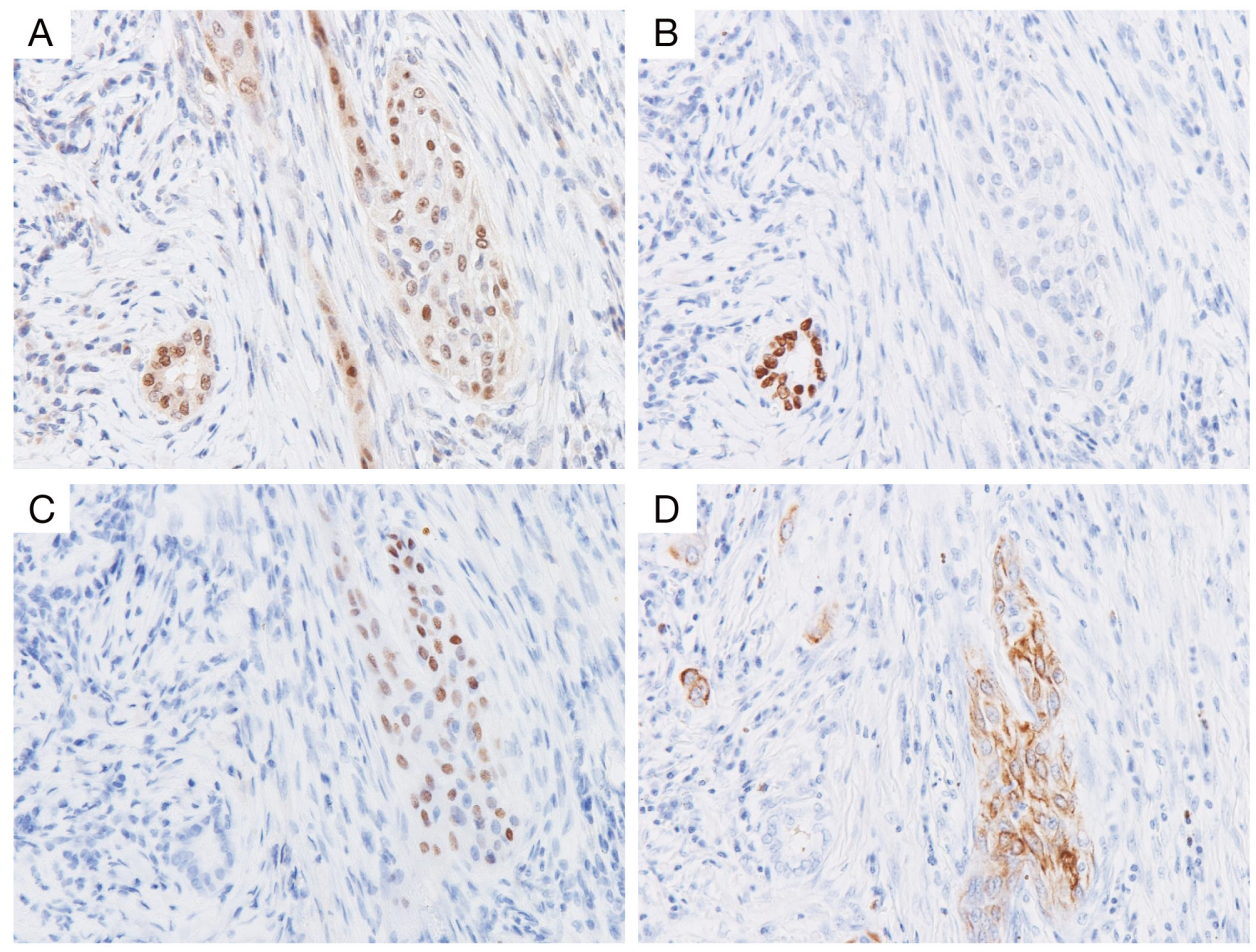

Fig. 2 Papillary carcinoma (left) associated with squamous metaplasia (right). Papillary carcinoma cells were positive for PAX8 (A) and TTF-1 (B), and negative for p63 (C) and high-molecular-weight cytokeratin (D). Squamous metaplastic cells were positive for PAX8 (A), p63 (C) and high-molecular-weight cytokeratin (D), and negative for TTF-1 (B). 
Table 3 Immunohistochemical results of extrathyroidal squamous cell carcinomas

\begin{tabular}{lcccc}
\hline & PAX8 & TTF-1 & p63 & HMW-CK \\
\hline Uterine cervix & $0(0 \%)$ & $0(0 \%)$ & $5(100 \%)$ & $5(100 \%)$ \\
Esophagus & $0(0 \%)$ & $0(0 \%)$ & $5(100 \%)$ & $5(100 \%)$ \\
Lung & $0(0 \%)$ & $0(0 \%)$ & $5(100 \%)$ & $5(100 \%)$ \\
\hline
\end{tabular}

TTF-1, thyroid transcription factor-1; HMW-CK, high-molecularweight cytokeratin.

Morula, ATC, and MEC did not react with TTF-1.

P63 and HMW-CK showed positive reactions to SCC, with the foci of squamous metaplasia and MEC revealing squamous differentiation (Figs. 1C, D, 2C, D). In SCC cases, p63 reacted with carcinoma cells principally located at the periphery of the tumor nests. Keratinizing carcinoma cells were more likely to be negative for the antibody. In contrast, HMW-CK was mainly positive for keratinizing or well-differentiated carcinoma cells. The number of HMW-CK-positive carcinoma cells was frequently higher than that of p63-positive carcinoma cells.

\section{Extrathyroidal carcinomas}

All uterine cervical, pulmonary, and esophageal SCC cases were negative for PAX8 and TTF-1. P63 and HMW-CK were strongly positive (Table 3 ).

\section{Discussion}

PAX8 is known to control the development of the central nervous system, eye, kidney, thyroid gland, and organs derived from the Müllerian duct [4]. In normal tissue, thyroid follicular cells, renal tubular epithelial cells, and epithelial cells of the fallopian tubes, endometrial glands, epididymis, vas deferens, and seminal vesicles have been shown to express PAX8 [4]. Furthermore, tumors arising in the Müllerian organs, kidney, and thyroid were found to maintain the expression of PAX8 [4].

In surgical pathology, the study of PAX8 has been limited. Nonaka et al. demonstrated that PTC, FTC, and PDTC invariably expressed PAX8 [5]. The expression of PAX8 was also detected in metastatic lesions [4]. Previous studies reported that PAX8 was mostly positive for ATC $[5,6]$. Bishop et al. reported that PAX8 staining was a reliable means of discerning a thyroid origin for undifferentiated tumors of the head and neck [6]. They demonstrated that all squamous ATC cases expressed PAX8. However, whether pri- mary thyroid SCC reacts with PAX8 has not yet been determined. Regarding the expression of PAX8 in PTC and ATC, our results were similar to previous findings. We demonstrated that the squamous metaplasia component associated with PTC, MEC, and primary SCC of the thyroid, which showed squamous differentiation, were also positive for PAX8.

Primary thyroid SCC expresses PAX8. Our results showed that SCC of the uterine cervix, esophagus, and lung did not react with PAX8. Ozcan et al. reported that SCC of the uterine cervix, lung, larynx, and skin did not express PAX8 [4]. SCC of the esophagus, lung, larynx, and skin do not express PAX8 because these organs do not naturally have PAX8 [4]. SCC of the uterine cervix and thyroid, both of which are organs expressing PAX8, showed different results; the former were negative while the latter positive. These results demonstrated that PAX8 staining was useful for distinguishing between primary thyroid SCC and invasion or metastasis from extrathyroidal SCC, especially in preoperative fine needle aspiration cytology or core needle biopsy, which cannot examine throughout the carcinoma.

In our study, differences were noted in the expression of PAX8 between cribriform variant PTC and conventional PTC. The latter invariably showed diffuse and strong positivity for the antibody. Staining of the former was weak and inhomogeneous. Only two PAX8-negative PTC cases were the cribriform variant. Squamous metaplasia was positive for PAX8, while the PAX8-positive rate of morules, which are characteristic of cribriform variant PTC and superficially similar to squamous metaplasia, was $14.3 \%$. The results of the present study support morules not being an early form of squamous metaplasia [8].

As with PAX8, TTF-1 is also a transcription factor. TTF-1 was previously shown to be a transcriptional regulator of thyroid-specific genes, and is also involved in the activation of lung-specific differentiation-inducing genes [9]. In normal tissue, thyroid follicular cells, alveolar epithelial cells type II, and Clara cells were found to be positive for TTF-1 staining [10]. In carcinomas, PTC, FTC, and pulmonary adenocarcinoma generally exhibited immunoreactivity for the antibody $[10,11]$. Therefore, TTF-1 has been immunohistochemically used as a marker to indicate thyroid or lung carcinomas [9]. PDTC often show decreased and focal staining for TTF-1, and most ATC lack TTF-1 reactivity $[11,12]$. On the other hand, PAX8 is even expressed in ATC and SCC, which may be explained 
by thyroid organogenesis. In view of the chronological emergence of transcription factors, PAX8 was identified as the earliest transcription factor, followed by TTF-1 [5]. Therefore, we considered the expression of PAX8 to be retained, even in PDTC, ATC, and SCC.

We herein clearly demonstrated that PAX8 was immunohistochemically positive for primary thyroid SCC and negative for extrathyroidal SCC. Therefore, PAX8 staining is considered useful for distinguishing between primary thyroid SCC and invasion or metastasis from extrathyroidal SCC. We recommend an immunohistochemical panel using PAX8 and TTF-1 to confirm a diagnosis of primary thyroid carcinoma. The expression of PAX8 may exclude TTF-1-positive pulmonary adenocarcinoma, whereas that of TTF-1 may exclude PAX8-positive carcinomas of the kidney and female genital tracts.

\section{Acknowledgments}

The authors thank Dr. Shigeo Yokoyama, Department of Diagnostic Pathology, Faculty of Medicine, Oita University, and Ms. Satomi Hoshikawa, Department of Pathology, Gunma University Hospital, for their comments on this manuscript.

\section{Disclosure}

None of the authors have any potential conflicts of interest associated with this research.

\section{References}

1. Lam KY, Sakamoto A (2004) Squamous cell carcinoma. In: Delellis RA, Lloyd RV, Heitz PU, et al. (ed) World Health Organization Classification of Tumors, Pathology and Genetics of Tumors of Endocrine Organs. IARC Press, Lyon, France: 81-82.

2. Thompson LDR (2012) Rare Primary Thyroid Epithelial Tumors. In: Nikiforov YE, Biddinger PW, Thompson LDR (ed) Diagnostic Pathology and Molecular Genetics of the Thyroid: A Comprehensive Guide for Practicing Thyroid Pathology. Lippincott Williams \& Wilkins, Philadelphia, USA: 286-289.

3. Barnes L (2004) Neuroendocrine tumors. In: Barnes L, Eveson JW, Reichart P, et al. (ed) World Health Organization Classification of tumors, Pathology and Genetics, Head and Neck Tumors. WHO Press, Geneva, Switzerland: 135-139.

4. Ozcan A, Shen SS, Hamilton C, Anjana K, Coffey D, et al. (2011) PAX 8 expression in non-neoplastic tissues, primary tumors, and metastatic tumors: a comprehensive immunohistochemical study. Mod Pathol 24: 751764.

5. Nonaka D, Tang Y, Chiriboga L, Rivera M, Ghossein R (2008) Diagnostic utility of thyroid transcription factors Pax8 and TTF-2 (FoxE1) in thyroid epithelial neoplasms. Mod Pathol 21: 192-200.

6. Bishop JA, Sharma R, Westra WH (2011) PAX8 immunostaining of anaplastic thyroid carcinoma: a reliable means of discerning thyroid origin for undifferentiated tumors of the head and neck. Hum Pathol 42: 18731877.

7. Hammond ME, Hayes DF, Dowsett M, Allred DC, Hagerty KL, et al. (2010) ASCO-CAP guideline recommendations for immunohistochemical testing of estrogen and progesterone receptors in breast cancer. J Clin Oncol 28: 2784-2795.

8. Hirokawa M, Kuma S, Miyauchi A, Qian ZR, Nakasono $M$, et al. (2004) Morules in cribriform-morular variant of papillary thyroid carcinoma: Immunohistochemical characteristics and distinction from squamous metaplasia. APMIS 112: 275-282.

9. Hammae SP, Dacic S (2013) Immunohistology of Lung and Pleural Neoplasms. In: Dabbs DJ (ed) Diagnostic Immunohistochemistry. Elsevier Health Sciences, Amsterdam, Netherlands: 398.

10. Moldvay J, Jackel M, Bogos K, Soltesz I, Agocs L, et al. (2004) The role of TTF-1 in differentiating primary and metastatic lung adenocarcinomas. Pathol Oncol Res 10:85-88.

11. Fabbro D, Loreto CD, Beltrami CA, Belfiore A, Di Lauro R, et al. (1994) Expression of Thyroid-specific Transcription Factors TTF-1 and PAX-8 in Human Thyroid Neoplasms. Cancer Res 54: 4744-4749.

12. Fischer S, Asa SL (2008) Application of Immunohistochemistry to Thyroid Neoplasms. Arch Pathol Lab Med 132: 359-372. 